Z. klin. Chem. u. klin. Biochem.

9. Jg., S. 367-374, September 1971

\title{
Isolierung eines kleinmolekularen Erythrocytenmembran-Proteins durch Anti D-Immunadsorption')
}

\author{
Von H. WeIcker und J. Metz \\ Aus der S. J. Thannbauser-Abteilung für Stoffwechseluntersucbungen \\ (Vorstand: Prof. Dr. H. Weicker) der Medizinischen Universitäts-Poliklinik Heidelberg
}

(Eingegangen am 6. Januar 1971)

\begin{abstract}
Nach Hämolyse gewaschener Rh-positiver Erythrocyten und anschließender erschöpfender Dialyse gegen dest. Wasser wurde das Hämolysat lyophilisiert, homogenisiert und dialysiert. Aus diesem Diffusat konnte durch Sephadex-Gelfiltration über G 10 eine konstant reproduzierbare kleinmolekulare Proteinfraktion isoliert werden. Durch Immunadsorption an gereinigtem Anti D, gekoppelt mit PABIonenaustauschercellulose wurde eine Substanz erhalten, deren Proteincharakter durch das IR-Spektrum und den Stickstoffgehalt von $14,6 \%$, die Biuretprobe und die Amidoschwarzanfärbung bewiesen wurde. Das Molekulargewicht lag um 10000. Ein Kohlenhydratanteil konnte nicht nachgewiesen werden. Lecithin, Cholesterin und Triglyceride lagen nicht vor. Eine geringe Konzentration von Lipidphosphor war nachweisbar. In chromatographischen und elektrophoretischen Untersuchungen ergab sich ein einheitliches Protein; nach salzsaurer Hydrolyse waren 14 verschiedene Aminosäuren dünnschichtchromatographisch nachweisbar. Aufgrund der gaschromatographischen Aminosäurenanalysen und Untersuchungen mit dem Aminosäureanalyser fanden wir, daß Lysin die höchste Konzentration der basischen, Asparaginsäure der sauren und Alanin der neutralen Aminosäure hatte. Es unterschied sich aufgrund seines Molekulargewichtes und seiner Aminosäurezusammensetzung bei hohem Lysingehalt von anderen bekannten Serum- und Erythrocytenmembranproteinen. Die Antigen-Charakterisierung wurde mit dem Schultz-Dale-Test durchgeführt; über die Ergebnisse dieser Untersuchungsreihe soll in einer zweiten Mitteilung berichtet werden.
\end{abstract}

\section{Isolation of a low molecular weight erythrocyte membrane protein by anti $D$ immunoadsorption}

Washed Rh-positive erythrocytes were haemolysed and the haemolysate was dialysed exhaustively against distilled water, followed by lyophilisation, homogenisation and dialysis. A constantly reproducible, low molecular weight protein fraction was isolated from the final dialysate by gel filtration on Sephadex G 10. Immunoadsorption on purified anti D, coupled with chromatography on PAB-ion exchange cellulose, gave a substance, which was shown to be a protein by its IR spectrum, nitrogen content of $14.6 \%$, biuret test and amidoblack staining. The molecular weight was about 10000 . There was no evidence for the presence of carbohydrate, lecithin, cholesterol or triglycerides. A very low concentration of lipid-bound phosphorus was detected. Chromatography and electrophoresis showed an homogeneous protein. After acid hydrolysis, 14 different amino acids were detected by thin layer chromatography.

Amino acids analysis by gas liquid chromatography and on the amino acid analyser showed that lysine, aspartic acid and alanine had the highest concentration of the basic, acidic and neutral amino acids, respectively. On the basis of molecular weight and an amino acid composition with a high concentration of lysine, the protein differs from other known proteins from the serum and erythrocyte membrane. The Schultz-Dale test was used for the antigen characterisation; the results from this series of experiments will be reported in a second communication.

Die zahlreichen Isolierungsversuche des Rh-Antigens, die im Anschluß an die serologische Charakterisierung des Rh-Faktors durch WIENER und LANDSTEINER 1940 ohne Erfolg vorgenommen wurden, zeigen, daß dieses Problem nicht einfach zu lösen ist. Sowohl die theoretisch zu erwartende minimale Antigen-Ausbeute als auch die Tatsache, daß bei den isolierten Substanzen durch Immunisierung im Tierversuch und immunologische Präzipitationsmethoden oder durch konventionelle serologische Verfahren keine eindeutigen Ergebnisse erhalten werden konnten, sind für diese unbefriedigenden Resultate mit verantwortlich $\mathrm{zu}$ machen. In den Untersuchungen, über die hier berichtet werden soll, erhielten wir ein kleinmolekulares Erythrocytenmembran-Protein, dessen Antigenfunktion nicht zerstört worden war. Da wir mit der SchultzDaLe-Technik D-Antigeneigenschaften an dem Protein, isoliert von $\mathrm{Rh}$-positiven Erythrocyten, nachweisen konnten und durch Anti D-Immunadsorbens eine

1) Mit Unterstützung der Deutschen Forschungsgemeinschaft. spezifische Isolierung vornahmen, soll die Frage, ob es sich bei diesem Protein um das Rh-Antigen D handelt, hier zur Diskussion gestellt werden. In dieser Mitteilung wird über die angewandte Isolierungstechnik und die biochemischen Eigenschaften des Membranproteins berichtet. Die Antigen-Charakterisierung mit der Schultz-Dale-Reaktion soll im Hinblick auf die zahlreichen methodischen Einzelheiten dieser Versuchsreihe in einer zweiten Mitteilung abgehandelt werden.

\section{Material und Methoden}

Insgesamt wurden 180 Konserven zu je $500 \mathrm{ml}$ gesondert aufgearbeitet. Die Konserven wurden uns von der Blutbank des Immunologischen und Serologischen Institutes der Universität Heidelberg zur Verfügung gestellt. Die Konserven wurden von dem Immunologischen und Serologischen Institut nach ihren $A, B, 0, M N$ - und Rh-Eigenschaften charakterisiert.

\section{Aufarbeitung}

Von $500 \mathrm{ml}$ Spenderblut wurden dic Erythrocyten durch Zentrifugation bei $7000 \mathrm{~g}$ von dem Plasma getrennt und mit physiolog. 
$\mathrm{NaCl}-\mathrm{Lösung}$ dreimal gewaschen. Nach diesem Waschvorgang war der Uberstand in der immunoelektrophoretischen Untersuchung frei von Plasmaproteinen. $200 \mathrm{ml}$ gepackte, gewaschene Erythrocyten wurden in 21 dest. Wasser $\mathrm{pH}$ 6,4 suspendiert und 24 Stdn. bei $4^{\circ}$ hämolysiert. Das Hämolysat wuzde in ViskingDialysierschläuchen $(\varnothing 20 \mathrm{~mm}) 3-4$ Tage in dest. Wasser bei $4^{\circ}$ dialysiert, bis der Schlauchinhalt kein freies $\mathrm{Na}^{+}, \mathrm{K}+$ oder $\mathrm{Ca}++$ mehr enthielt. Das Hämolysat wurde gefriergetrocknet, das Lyophylisat zu einer wäßr. 20proz. Suspension homogenisiert und erneut gegen dest. Wasser dialysiert. Nach $48 \mathrm{stdg}$. Dialyse mit mehrfachem Wechsel der Dialyseflüssigkeit wurde das Diffusat (I) lyophilisiert. Von dem nichtdiffusiblen Material wurde nach 24 stdg. Stehen bei $4^{\circ}$ der Überstand durch Zentrifugation bei $7000 \mathrm{~g}$ vom Bodensatz getrennt. Dex Uberstand wurde gefriergetrocknet und nochmals $24 \mathrm{Stdn}$. gegen dest. Wasser dialysiert. Das lyophilisierte Diffusat (II) wurde mit dem Diffusat (I) vereinigt und über Sephadex G 10 gelfiltriert. Säule $100 \times 0,8 \mathrm{~cm}$, Auftragsmenge $150-200 \mathrm{mg}$, Diffusat I/II, gelöst in $0,5-0,8 \mathrm{ml}$ dest. Wasser. Die Fraktionen wurden mit dest. Wasser bei einer Fließgeschwindigkeit von $60 \mathrm{ml} / \mathrm{Std}$. in Einzelportionen von $1,5 \mathrm{ml}$ eluiert und mit dem Uvicord bei $280 \mathrm{~nm}$ registriert. Die einzelnen Fraktionen, ermittelt nach der Registrierung bei $280 \mathrm{~nm}$, wurden bei $20^{\circ}$ im Vakuumrotationsverdampfer eingeengt und anschließend lyophilisiert. Aus der Fraktion I, die die stärkste UV-Absorption zeigte, wurde durch Elution von Anti-D-Immunadsorbens eine elektrophoretisch homogene Proteinkomponente isoliert (Abb. 1).

\section{Immunadsorptionsverfahren}

Von den auf ihre Leistungsfähigkeit untersuchten Immunadsorbensverfahren hat sich die Methode mit Anti D-Kopplung auf PAB-Ionenaustauschercellulose durch Diazotierung (1) besser bewährt als die Diazotierung von Polyaminostyrol (2) oder die Glutaraldehydbehandlung (3) von DEAE-Cellulose. Hierzu wurden $2 \mathrm{~g}$ PAB-Cellulose in $50 \mathrm{ml} 2 \mathrm{~N} \mathrm{HCl}$, versetzt mit $10 \mathrm{ml}$ 14proz. Natriumnitrit, suspendiert. Diese Suspension wurde $1 \mathrm{Std}$. bei $0^{\circ}$ gerührt, das Reaktionsprodukt anschließend auf einem Büchnertrichter abfiltriert und mit 21 dest. Wasser nachgewaschen. Nach diesem Waschvorgang wurde die PAB-Cellulose in $200 \mathrm{ml}$ 5proz. Natriumacetat-Harnstofflösung gerührt, abfiltriert und mit 21 dest. Wasser nachgewaschen. Nach nochmaligem Filtrieren auf einem Büchnertrichter wirde die diazotierte PAB-Cellulose mit $2 \mathrm{ml}$ gereinigtem Anti $\mathrm{D}$ in $10 \mathrm{ml}$ physiolog. $\mathrm{NaCl}-\mathrm{Lösung}$, Titer 1:256 $4 \mathrm{Stdn}$. bei $0^{\circ}$ unter leichtem Rühren gekoppelt. Nach Zentrifugation war der Uberstand Anti D-frei. Der Bodensatz wurde mehrfach mit physiolog. $\mathrm{NaCl}$-Lösung nachgewaschen und in $11 \beta$-Naphthollösung suspendiert, $(\beta$-Naphthollösung: $1 \mathrm{~g} \beta$-Naphthol wird in $110,1 \mathrm{~N} \mathrm{NAOH}$ gelöst, der pH-Wert wurde mit 8,5proz. Orthophosphorsäure auf pH 8 eingestellt, und nach 24 Stdn. bei $+4^{\circ}$ filtriert), und über Nacht unter ständigem Rühren bei $+4^{\circ}$ aufbewahrt. Dabei wird $\beta$-Naphthol an die nicht durch Anti D besetzten Diazogruppen gekoppelt. Anschließend wurde die überschüssige $\beta$-Naphthollösung entfernt und der Bodensatz mit 11 physiolog. NaCl-Lösung nachgewaschen. Das Anti-D-Immunadsorbens wurde 2 Tage gegen dest. Wasser bei $+4^{\circ}$ dialysiert. Das Anti D-IgG-Serum wurde uns von dem Immunologischen und Serologischen Institut zur Verfügung gestellt. Es war durch das von ROELCKE und JUNGFER (4) ausgearbeitete Verfahren einer Hydroxylapatit-Säulenchromatographie boch konzentriert. Nach dieser Vorbereitung des Immunadsorbens wurde die Anti D-tragende PAB-Cellulose mit der Fraktion I des gelfiltrierten Diffusates I/II unter leichtem Rühren 1 Std. bei $37^{\circ}$ inkubiert. Danach wurde das inkubierte Immunadsorbens in ein temperierbares Chromatographierohr eingefüllt und bei $37^{\circ}$ zunächst so lange mit dest. Wasser gewaschen, bis keine UV-absorbierenden Substanzen mehr eluiert werden konnten. Das Eluat wurde mit dem Uvicord bei $280 \mathrm{~nm}$ registriert und im Fraktionssammler gesammelt; nach Einengung im Rotationsverdampfer bei $20^{\circ}$ wurde es gegen dest. Wasser $\mathrm{pH} 6,4$ dialysiert und das Diffusat gefriergetrocknet. Der Säuleninhalt wurde mit $50 \mathrm{ml}$ dest. Wasser nachgewaschen, anschließend bei $20^{\circ} \mathrm{mit}$ $0,1_{\mathrm{M}}$ Glycinpuffer bei $\mathrm{pH} 2,5$ eluiert. Die Elutionsgeschwindigkeit betrug $30 \mathrm{ml} / \mathrm{Std}$. bei Einzelportionen von 1,0 ml. Die Registrierung erfolgte bei $280 \mathrm{~nm}$. Die proteinhaltige Fraktion wurde gefriergetrocknet und das Protein vom Glycinpuffer über Sephadex G 10 abgetrennt. Die Identifizierung der glycinfreien Proteinfraktion erfolgte mit der quantitativen Ninhydrin-Reaktion in den einzelnen Reagenzgläsern des Fraktionssammlers (Tab. 1).

Tab. 1

Isolierung des Anti D-hemmenden klẹipmolekularen Proteins

Abtrennung der Erythrocyten aus $500 \mathrm{ml}$ Konservenblut durch Zentrifugation bei $7000 \mathrm{~g}$

Waschen der Erythrocyten mit 0,9proz. NaCl-Lösung

Hämolyse von $180 \mathrm{ml}$ gepackter Erythrocyten in 21 dejst. Wasser bei pH 6,4 für 24 Stdn. bei $4^{\circ}$ Dialyse des Hämolysates in Visking-Dialysierschläuchen $2 / 22$ gegen
dest. Wasser $\mathrm{pH} 6,4$ für 3 Tage bei $4^{\circ}$

Gefriertrockung des dialysierten Hämolysates. Ausbeute: 50-60 g Homogenisieren des lyophilisierten Hämolysates zu 20proz. wäßr. Suspension

Dialyse des Homogenisates gegen dest. Wasser pH 6,4 für 2 Tage bei 4

Einengen des Diffusates I im Rotationsvakuumverdampfer bei $25^{\circ}$ und Gefriertrocknung. Ausbeute: $150 \mathrm{mg}$

Abtrennung des Stromas im nichtdiffusiblen Material nach 24stdg. Stehen bei $4^{\circ}$ und Gefriertrocknung

Dialyse des Uiberstandes nach Homogenisieren gegen dest. Wasser pH 6,4 24 Stdn. bei $4^{\circ}$

Einengen des Diffusates II im Rotationsvakuumverdampfer bei $25^{\circ}$ Gefriertrocknung. Ausbeute: $20-30 \mathrm{mg}$

Gelfiltration des Diffusates $I$ und II an Sephadex G 10, Säulenlänge $1000 \mathrm{~mm}$, Säulenweite $8 \mathrm{~mm}$, Elution mit Wasser

Immunadsorption der Fraktion I nach Sephadex-Gelfiltration an gereinigtem Anti D gekoppelt mit PAB-Cellulose durch Diazotierung. Absprengung mit $0,1 \mathrm{M}$ Glycinpuffer bei $\mathrm{pH} 2,5$ nach vorausgegangener
Elution des. Immunadsorbens mit Wasser bei $37^{\circ}$. Ausbeute: $3-4 \mathrm{mg} /$ 1 Blut.

\section{Homogenitätsprüfung}

Refiltration des Diffusates I/II über Sephadex-G 10-Säule $30 \times 0,8 \mathrm{~cm}$, Elutionsgeschwindigkeit $60 \mathrm{ml} / \mathrm{Sta}$. in Einzelportionen zu 1,5 ml. Registrierung bei $280 \mathrm{~nm}$. Die Fraktionen wurden gesammelt, eingeengt und lyophilisiert.

Ionenaustauscher-Chromatographie des Diffusates I/II an DEAECellulose, Säule $30 \times 0,8 \mathrm{~cm}$, Arbeits-Kapazität $.52 \mathrm{mVal}$, stufenweise Elution mit Trispuffer nach Äquilibrierung des Ionenaustauschers mit $0,01 \mathrm{M}$ Trispuffer. Die Zusammensetzung des $0,2 \mathrm{M}$ Tris-Stammpuffers $\mathrm{pH} 8,0$ ist folgende: $6,057 \mathrm{~g}$ Tris $+134 \mathrm{ml}$ $0,2 \mathrm{~N} \mathrm{HCl}$ ad 11 dest. Wasser; bei konstantem Puffer-pH wurde mit $0,001,0,01$ und $0,1 \mathrm{M}$ Trispuffer eluiert.

Cbromatograpbie des Diffusates I/II auf Cellulose-Dünnschichtplatten der Firma Merck $20 \times 20 \mathrm{~cm}$. Trenntechnik eindimensional bei $17^{\circ}$ Raumtemperatur. Laufmittel a) Butanol: Eisessig: Wasser $=$ 120:30:50 ( $/ \mathrm{v} / \mathrm{v} / \mathrm{v})$, Laufmittel b) t-Butanol: Methyläthylketon: Ameisensäure: Wasser $=3: 6: 3: 3(\mathrm{v} / \mathrm{v} / \mathrm{v})$. Im Laufmittel $\mathrm{b}$ erfolgte zweimalige Entwicklung der Dünnschichtplatte.

Ziveidimensionale Chromatographie auf Celluloseplatten $7,5 \times 7,5 \mathrm{~cm}$. I. Dimension Butanol:Eisessig: Wasser $=120: 30: 5(\mathrm{v} / \mathrm{v} / \mathrm{v})$; II. Dimension Phenol: Wasser:Ammoniak $=90: 36: 0,9(\nabla / v / v)$. Die Identifizierung erfolgte mit 0,25 proz. Ninhydrin in Aceton und mit Chlor-Tolidinsprühreagenz für Peptide (5). Die eindimensionale Chromatographie für Lipidsubstanzen wurde auf Kieselgel H-Platten durchgeführt. Laufmittel Chloroform:Methanol: Wasser $=65: 25: 4(\mathrm{v} / \mathrm{v} / \mathrm{v})$. Identifizierung der Phosphatide erfolgte mit dem Reagenz nach Zinzadze (6). Cholesterin, Phosphatidund Fettsäurennachweis wurde mit Joddampf oder mit Antimon(V)-Chlorid-gesättigter Chloroformlösung vorgenommen.

\section{Elektrophorese-Technik}

a) Fingerprint auf Kieselgel H-Dünnschichtplatten $20 \times 20 \mathrm{~cm}$, I. Dimension, Hochspannungselektrophorese (Gerät Fa. Hormuth). Puffersystem 1: Pyridin: Essigsäure: Wasser $=100: 10: 890(\mathrm{v} / \mathrm{v} / \mathrm{v})$, pH 6,5. $90 \mathrm{Min}$. bei $0^{\circ}, 1 \mathrm{kV}, 36 \mathrm{~mA}$. Puffersystem 2: Essigsäure: Ameisensäurè: Wasser $=50: 150: 850(\nabla / v / v), \mathrm{pH} 1,9.90 \mathrm{Min}$. bei $1^{\circ}, 960$ Volt $43 \mathrm{~mA}$. II. Dimension, Chromatographie: Lauf- 
mittel Butanol:Eisessig: Wasser 4:1:5 (v/v/v), $14 \mathrm{Stdn}$. bei $+5^{\circ}$. Nachweis mit 0,25proz. Ninhydrin in Aceton und Chlor-Tolidinsprühreagenz.

b) Cellulose-Acetatfolien-Elektrophorese auf Membranfolien der Fa. Schleicher \& Schüll, Trennung bei 250 Volt, 3-4 mA. Natrium-Veronalpuffer pH 8,6 in der Beckman Mikroelektrophorese-Kammer, Anfärbung mit Ponceau-Rot oder Amidoschwarz B.

c) Disk-Elektrophorese auf 7,5proz. Polyacrylamid, pH 8,9, Sammel- und Trenngel-Technik (Gerät der Fa. Shandon) Trisphosphatpuffer $\mathrm{pH} 8,9$ Trennung bei $150 \mathrm{~V}, 3 \mathrm{~mA}$ pro Gelzylinder. Anfärbung mit Amidoschwarz B in 7proz. Essigsäure. Entfärbung mit 7proz. Essigsäure (7).

\section{Kolorimetrische Untersuchungen}

\section{Koblenbydrate}

Die Hexosen wurden nach der Orcin-Methode (8), die Pentosen nach Dische und Shetrles (9), die Neuraminsäure nach SvennerHOLM (10) und WARREN (11), die Hexosamine nach CESSI und Mitarbeitern (12) bestimmt.

\section{Lipide}

Die Phosphatide wurden nach Fiske und Subarrow (13) ermittelt. Diese Methode wirde modifiziert durch Erhitzen der Proben nach Farbentwicklung für 7 Min. bei $100^{\circ}$. Dic Cholesterinbestimmung wurde nach Pearson, Stern und Mitarbeitern (14) durchgeführt.

\section{Proteine}

Der Eiweißstickstoff wurde nach KJELDAHL (15) ermittelt, die Peptid- und Aminosäure-Bestimmung wurde mit der BiuretMethode und der quantitativen Ninhydrin-Reaktion ausgeführt. Die Ninhydrin-Reaktion wurde folgendermaßen vorgenommen: Lösung a) $21,008 \mathrm{~g}$ Citronensäure wurden in $20 \mathrm{ml} 1 \mathrm{~N} \mathrm{NaOH}$ gelöst und auf $50 \mathrm{ml}$ mit dest. Wasser aufgefüllt, $\mathrm{pH} \mathrm{5,0}$. Hierzu wurden 0,8 g Zinn-[II]-chlorid hinzugegeben.

Lösung b) $20 \mathrm{~g}$ Ninhydrin wurden in $50 \mathrm{ml}$ Äthylenglykolmonomethyläther gelöst. Ausführung: Lösung a und b wurden vor Gebrauch im Verhältnis $1: 1$ gemischt. $0,1 \mathrm{ml}$ Probe und $1 \mathrm{ml}$ Ninhydrin-Reagenzgebrauchslösung wurden $20 \mathrm{Min}$. bei $100^{\circ}$ erhitzt. Anschließend wurden $5 \mathrm{ml}$ Propanol: Wasser in einem Mischungsverhältnis $1: 1(\mathrm{v} / \mathrm{v})$ hinzugesetzt, die Probe $20 \mathrm{Min}$. im Dunkeln aufbewahrt und die Extinktionen bei $570 \mathrm{~nm}$ gemessen.

\section{Hydrolyse und Chromatographie}

\section{Aminosäuren}

$1 \mathrm{mg}$ Substanz wurde in $2 \mathrm{ml} 6 \mathrm{~N} \mathrm{HCl} 24 \mathrm{Stdn}$. bei $100^{\circ}$ hydrolysiert. Die qualitative Aminosäurebestimmung wurde mit der zweidimensionalen Dünnschichtchromatographie auf Celluloseplatten 7,5 $\times 7,5 \mathrm{~cm}$ (s. S. 368) durchgeführt. Die quantitative Aminosäurebestimmung wurde gaschromatographisch nach der Methode von GEHRKE und Mitarbeitern $(16,17)$ vorgenommen. Hierbei wurde das trockene Hydrolysat nach Zugabe von $50 \mu \mathrm{g}$ Myristinsäure als internem Standard in $1,25 \mathrm{mVal} \mathrm{HCl}$ in $\mathrm{Me}$ thanol bei Raumtemperatur zu den Aminosäure-Methylesternderivatisiert, danach folgte die Umesterung zu den AminosäureButylestern mit $1,25 \mathrm{mVal} \mathrm{HCl}$ in n-Butanol $150 \mathrm{Min}$. bei $100^{\circ}$. Die Trifluoracetylierung wurde mit $0,05 \mathrm{ml}$ Trifluoracetanhydrid und $0,05 \mathrm{ml}$ Methylenchlorid $150 \mathrm{Min}$. bei Raumtemperatur oder $5 \mathrm{Min}$. bei $150^{\circ}$ durchgeführt. $1-2 \mathrm{ml}$ wurden in den Gaschromatographen eingespritzt. Gaschromatographische Bedingungen: Glassäulen $180 \times 0,3 \mathrm{~cm}$, im Gerät Varian Aerograph 1800 .

Säulenfüllung A) $1,5 \%$ OV 17 auf Chromosorb G-HP 100 bis $120 \mathrm{mesh}$; Ofentemperatur: Anfangstemperatur $95^{\circ}$, Endtemperatur $200^{\circ}$, Temperaturprogramm $10 \mathrm{Min}$. Isotherm, $2^{\circ} / \mathrm{Min}$. bis Endtemperatur.

Säulenfüllung B) $0,625 \%$ EGA auf Chromosorb W/AW 80 bis 120 mesh, Ofentemperatur: Anfangstemperatur $80^{\circ}$, Endtemperatur $200^{\circ}$, Temperaturprogramm $4 \mathrm{Min}$. Isotherm $2^{\circ} / \mathrm{Min}$. bis Endtemperatur, $20 \mathrm{Min}$. Isotherm bei Endtemperatur.

\section{Koblenbydrate}

$5 \mathrm{mg}$ Substanz wurden in $2 \mathrm{ml} 2 \mathrm{~N}$ Schwefelsäure $4 \mathrm{Stdn}$. bei $100^{\circ}$ hydrolysiert. Nach Entfernung der Schwefelsäure als Bariumsulfat durch Zentrifugation und Ionenaustauscher-Passage über Dowex $50 \times 8$ (Säule $10 \times 0,8 \mathrm{~cm}$ in H+-Form) wurde die Dünnschichtchromatographie auf Celluloseplatten wie oben (s. S. 368) vorgenommen. Die Identifizierung der Neutralzucker erfolgte mit Anilinphthalat-Sprühreagenz nach Erhitzen auf $100^{\circ}$ für 5 Min. (18). Die quantitative Bestimmung der Neutralzucker und Hexosamine erfolgte nach der Methode von YANG und HAKOMORI

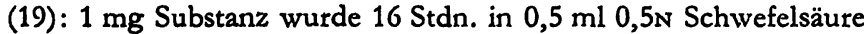
in 90proz. Essigsäure hydrolysiert. Anschließend wurde $0,5 \mathrm{ml}$ dest. Wasser zugegeben und erneut für $6 \mathrm{Stdn}$. bei $80^{\circ}$ hydrolysiert. Das Hydrolysat wurde über $40 \mathrm{mg}$ Dowex $1 \times 8$ in Acetatform passiert und der Ionenaustauscher mit $10 \mathrm{ml}$ Methanol nachgewaschen. Einengung des Eluates unter Stickstoff und Extraktion des Rückstandes in $2 \mathrm{ml}$ Wasser:Chloroform = $1: 1(v / v)$. Die Reduktion erfolgte in $8 \mathrm{M}$ wäßr. Natriumborhydridlösung, die Reaktionszeit betrug $2 \mathrm{Stdn}$. Danach wurde mit Essigsäure neutralisiert, und mit $3 \mathrm{ml}$ Metbanol:Eisessig = 200:1 (v/v) dreimal unter Stickstoff abgedampft und das Borat als Borsäure-Trimethylester vertrieben. Anschließend wurde das Präparat über Phosphorpentoxid im Exsikkator zur Trockene gebracht. $5 \mathrm{ml}$ Essigsäureanhydrid wurde zugesetzt und $2 \mathrm{Stdn}$. bei $100^{\circ}$ erhitzt. Nach Zugabe von $2 \mathrm{ml}$ Toluol wurde unter Stickstoff mehrfach abgedampft, bis das Essigsäureanhydrid vollkommen vertrieben war. Nach Trocknung wurden $2 \mathrm{ml}$ Chloroform: Wasser = 1:1 (v/v) zugesetzt, die Wasserphase wurde verworfen, die Chloroformphase auf $0,01 \mathrm{ml}$ eingeengt und ein Aliquot von 0,001 bis $0,002 \mathrm{ml}$ zur Gaschromatographie verwandt. Die Chromatographie wurde auf einer 3proz. ECNSS-MSäule $150 \times 0,3 \mathrm{~cm}$ vorgenommen. Säulenfüllung: Gaschrom Q 100-120 mesh, Temperaturprogramm: Ofentemperatur $180^{\circ}$ Isotherm $15 \mathrm{Min}$. , danach $2^{\circ} / \mathrm{Min}$. bis $210^{\circ}$ Endtemperatur. Mit dieser Technik konnten Pentosen, Hexosen, Glucosamin und Galaktosamin aufgetrennt werden.

Die Molekulargewichtsbestimmung wurde nach der Gelfiltrationsmethode unter Verwendung von Ribonuclease und Protaminsulfat als Referenz durchgeführt. Die IR-Spektren wurden von $\mathrm{KBr}$-Preßlingen mit dem Gerät von Perkin-Elmer aufgenommen.

\section{Antigen-Charakterisierung}

Die technischen Einzelheiten der Antigen-Charakterisierung sollen in einer zweiten Mitteilung ausführlich dargestellt werden.

\section{Ergebnisse}

Die Isolierung kleinmolekularer Proteine aus der Erythrocytenmembran wurde durch Hämolyse in dest. Wasser bei pH 6,4, Gefriertrocknung, Homogenisierung und Dialyse unter Vermeidung aggressiver Stromaaufspaltung vorgenommen und ergab bei 100 Vergleichsuntersuchungen gut reproduzierbare Werte (Tab. 1). Durch diese schonende Aufarbeitung wurde die Antigenfunktion des Proteins nicht zerstört. Die Gesamtausbeute des Diffusates I/II, gewonnen aus dem gefriergetrockneten und homogenisierten Hämolysat betrug $130-200 \mathrm{mg} / 200 \mathrm{ml}$ gepackte Erythrocyten. Durch Dialyse des nativen Hämolysates, die vor der Gefriertrocknung durchgeführt worden war, konnten $\mathrm{Na}^{+}, \mathrm{K}^{+}, \mathrm{Ca}^{+}+$, Aminosäuren und andere kleinmolekulare organische Substanzen der Erythrocytenmembran eliminiert werden (Abb. 1a). Durch Gelfiltration an Sephadex G 10 wurde das Diffusat I/II in mehrere Fraktionen aufgetrennt, von denen die erste aus kleinmolekularen Proteinen bestand (Abb. 2a). Wurde die Gelfiltration des Diffusates 


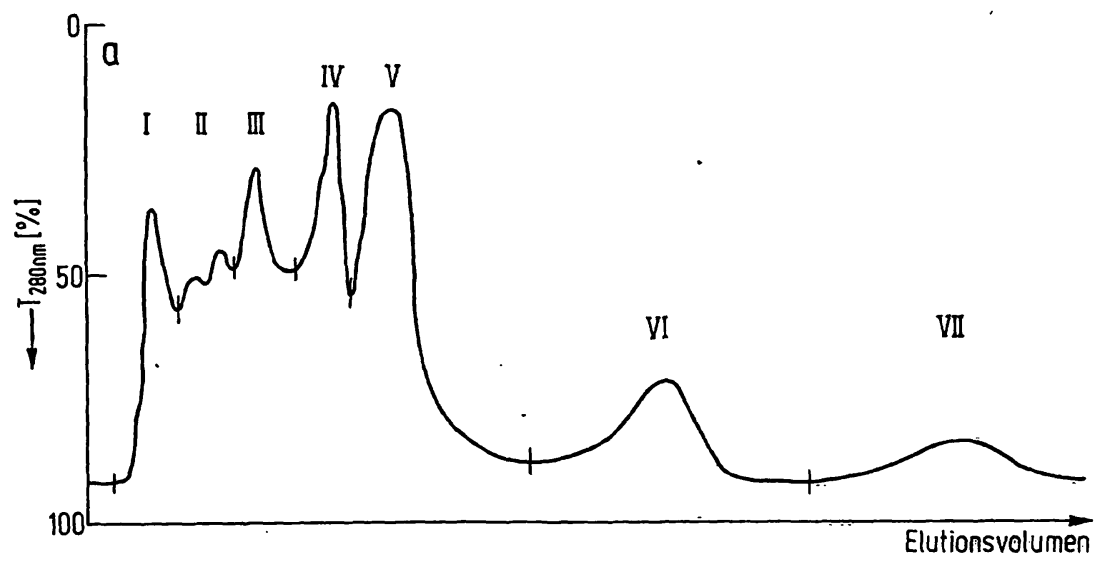

Abb. 1a und $b$

Gelfiltration über Sephadex-G 10-Säule $100 \times 0,8 \mathrm{~cm}$, Auftragsmenge $150 \mathrm{mg}$, gelöst in $0,5 \mathrm{ml}$ dest. Wasser. Elution mit dest. Wasser $60 \mathrm{ml} / \mathrm{Std}$. Registrierung Uvicord, $\lambda=280 \mathrm{~nm}$

a) Diffusat I/II des lyóphilisierten und homogenisierten Hämolysates von Rh-positiven Erythrocyten vor Dialyse des nativen Hämolysates. K̇onserve 151 , Blutgruppe $0 \mathrm{Rh}+$

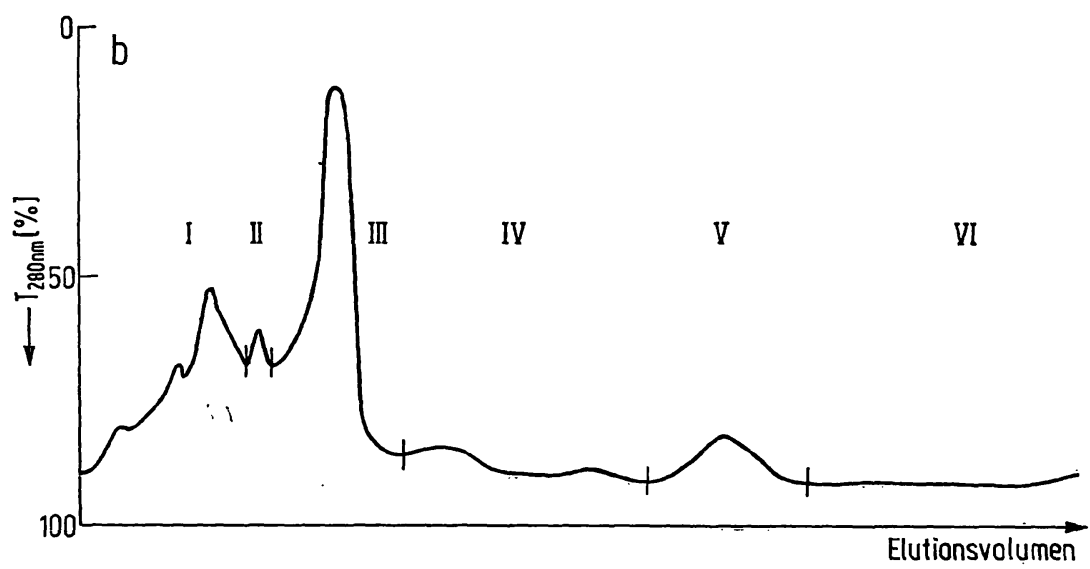

b) Diffusat $I / I I$ des Hämolysates von $R$ hpositiven Erythrocyten, ohne Gefriertrocknung und Homogenisieren des dialysierten nativen Hämolysates. Konserve 142, Blutgruppe A $\mathrm{Rh}+\mathrm{MN}$

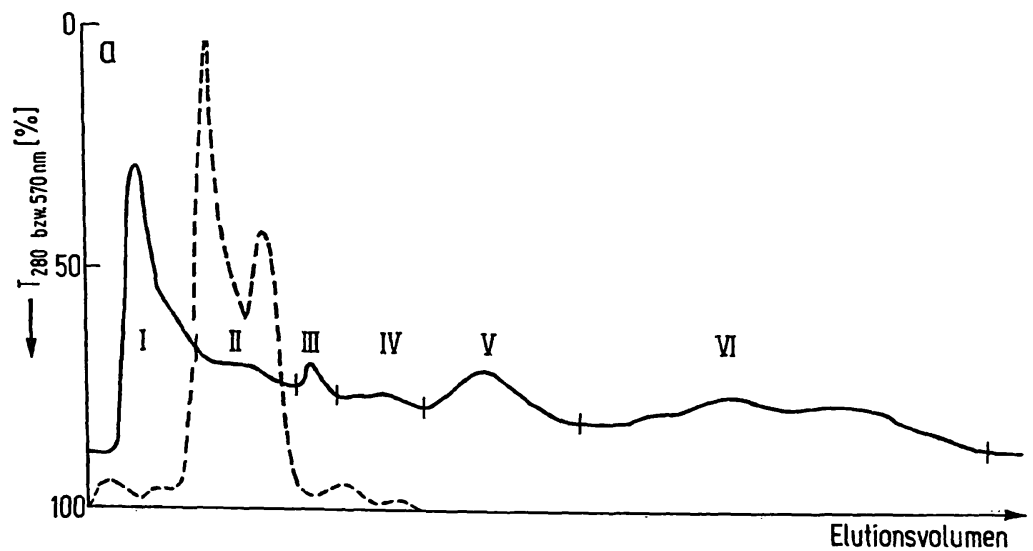

Abb. 2

Gelfiltration über Sephadex-G 10-Säule $100 \times 0,8 \mathrm{~cm}$, Auftragsmenge $150 \mathrm{mg}$, gèlöst in $0 ; 5 \mathrm{ml}$ dest. Wasser. Elution mit dest. Wasser $60 \mathrm{ml} / \mathrm{Std}$. Registrierung Uvicord, $\lambda=280 \mathrm{~nm}(\longrightarrow$ und Ninhydrin-Reagenz, $\lambda=570 \mathrm{~nm}(--)$

a) Diffusat I/II des lyophilisierten und homogenisierten Hämolysates von Rh-positiven Erythrocyten nach Vordialyse-des nativen Hämolysates. Konserve 163, Blutgruppe $0 \mathrm{Rh}+$ (ccDEe) $\mathrm{MN}$

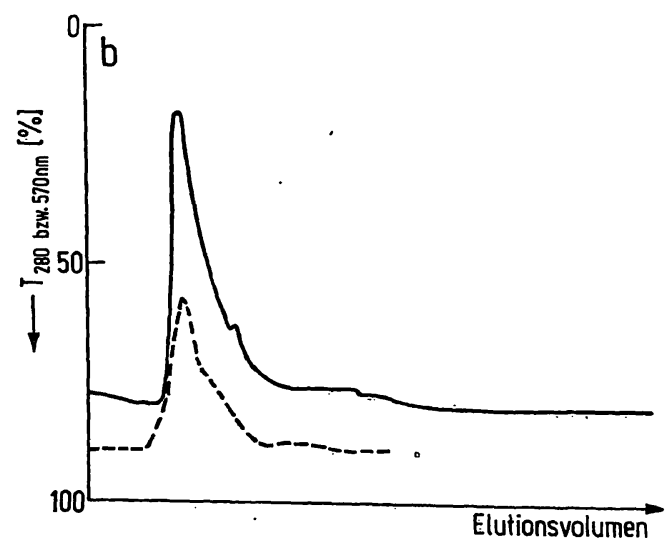

b) Rechromatographie der Fraktion I 


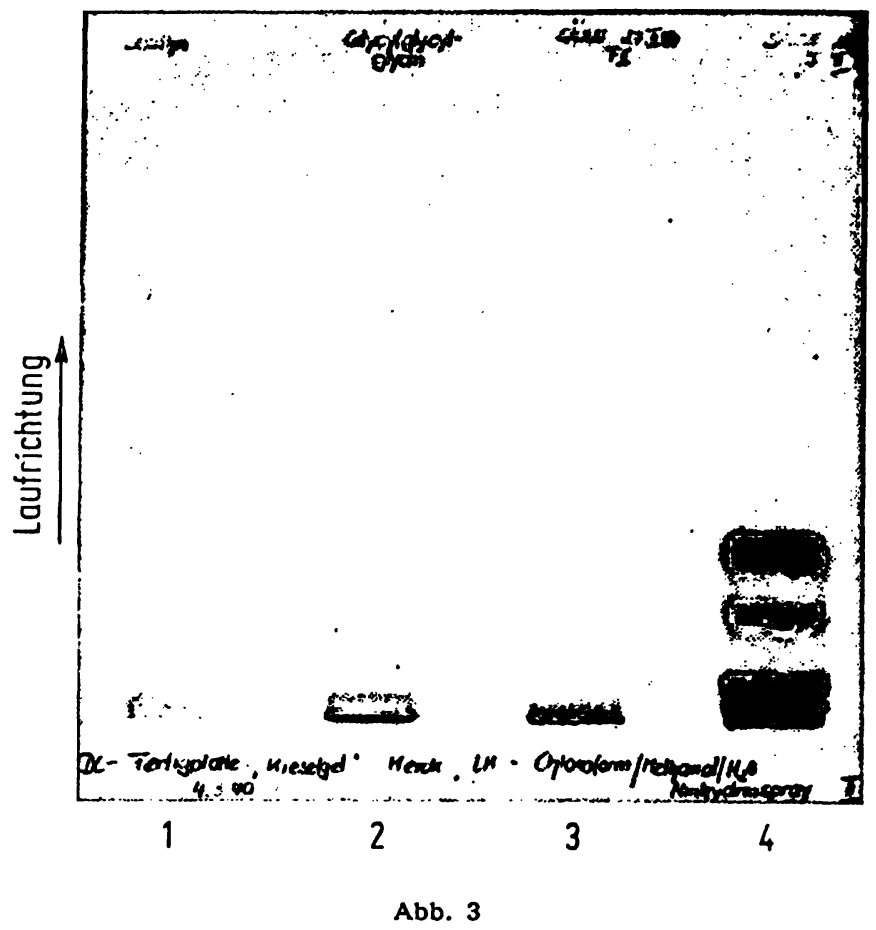

Dünnschicht-Chromatographie auf Fertigplatten Merck $20 \times 20 \mathrm{~cm}$, Laufmittel Chloroform: Methanol: Wasser $=65: 25: 4(\mathrm{v} / \mathrm{v} / \mathrm{v})$. 1. Le(t) 0,25 proz. Ninhydrin-Aceton-Sprühreagenz

ohne vorausgegangene Gefriertrocknung und Homogenisierung des Hämolysates durchgeführt, dann fehlte diese proteinhaltige Fraktion (Abb. $1 \mathrm{~b}$ ). Hieraus kann geschlossen werden, daß erst nach Entfernung der für die Membranstabilität wichtigen Mineralien und durch die nachfolgende physikalische Behandlung des Stromas diese Proteine aus dem Stromaverband freigesetzt wurden, so daß sie durch Dialyse isoliert werden konnten. Aus $200 \mathrm{mg}$ diffusiblem Material erhielten wir nach Gelfiltration und Refiltration über Sephadex G $1020-30 \mathrm{mg}$ der kleinmolekularen proteinhaltigen Fraktion I (Abb. 2a, b, Tab. 1). In der eindimensionalen Dünnschichtchromatographie auf Celluloseplatten war dieses Protein sowohl mit Ninhydrin als auch mit dem für Peptidnachweis verwendbaren Chlor-Tolidin-Sprühreagenz als eine Bande dargestellt. Die $R_{\mathrm{F}}$-Werte dieser Bande betrugen 0,05. In der zweidimensionalen Chromatographie war es in dem Laufmittel Butanol:Eisessig: Wasser und Phenol: Wasser:Ammoniak mit Ninhydrinsprühreagenz als ein Fleck zu identifizieren. Dieser Fleck zeigte ebenfalls nur eine geringe chromatographische Mobilität (Abb. 3, 4). Auf CelluloseacetatFolien und Polyacrylamid konnten nach Sephadex-G 10Refiltration elektrophoretisch noch 3 bzw. 6 Banden dargestellt werden. In dem Fingerprint-Verfahren zeigte diese Substanz nach hochspannungselektrophoretischer Trennung bei 1000 Volt ebenfalls noch 3 Flecke, die in der nachfolgenden dünnschichtchromatographischen Untersuchung jedoch keine Mobilität mehr aufwiesen.

Der Proteincharakter konnte mit der quantitativen Ninhydrin-Reaktion, die gegenüber dem gewichtsgleichen Aminosäurestandard eine vielfach geringere

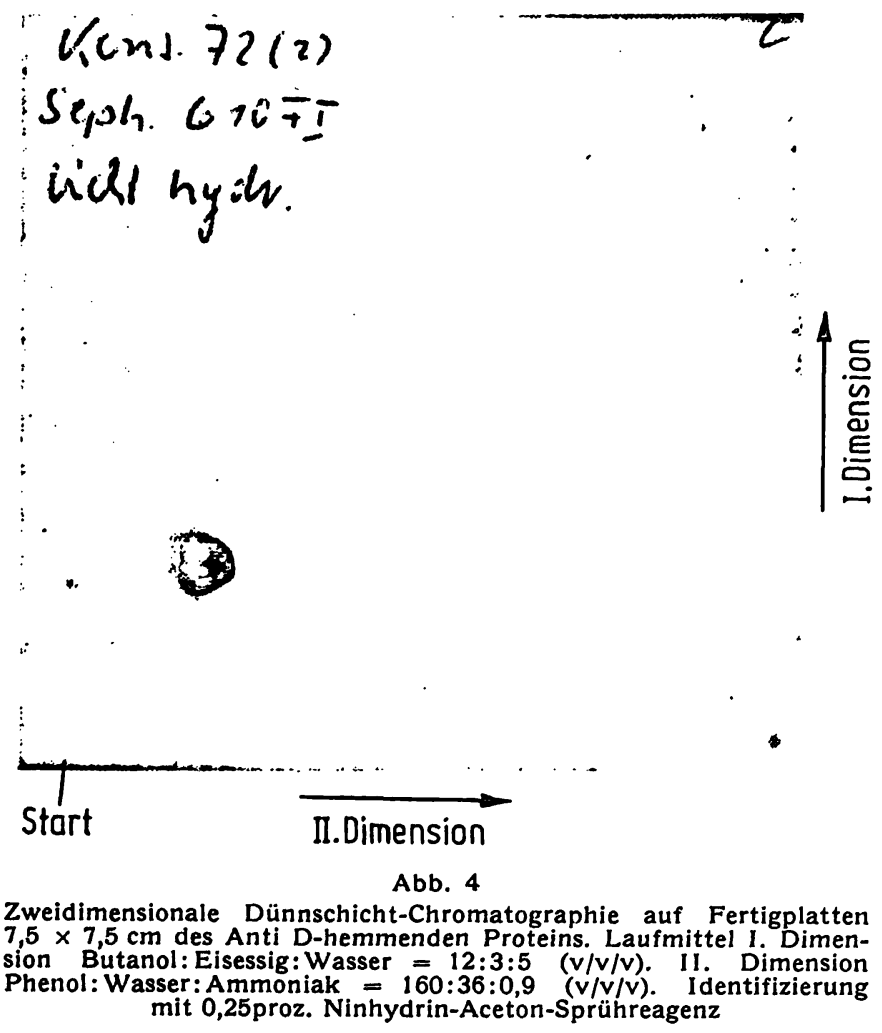

Intensität ergab, sowie durch die stark positive BiuretReaktion und Amidoschwarzfärbung wahrscheinlich gemacht werden. Das IR-Spektrum zeigte für Peptide charakteristische Banden. Der Stickstoffgehalt betrug 14,5\%. Der Proteingehalt lag um $90 \%$; Hexosen, Pentosen, Hexosamine und Neuraminsäure waren weder durch die oben erwähnten kolorimetrischen Methoden, durch die Dünnschichtchromatographie noch durch die gaschromatographischen Untersuchungen nach vorausgegangener Hydrolyse und nachfolgender Reduktion durch Natriumborhydrid und Acetylierung nachweisbar. Mit der modifizierten LipidPhosphor-Bestimmung nach FrSKe und SUBARrow fanden wir 0,5-1\% Lipidphosphor. Cholesterin oder Neutralfette waren nicht faßbar, flammenphotometrisch lagen die $\mathrm{Na}^{+}, \mathrm{K}^{+}$- und $\mathrm{Ca}^{++}$-Werte unter $0,01 \%$. $\mathrm{Da}$ die proteinhaltige Fraktion nach Gelfiltration an Sephadex G10 elektrophoretisch noch in mehrere Banden aufgetrennt werden konnte, führten wir eine Immunadsorption mit gereinigtem Anti $\mathrm{D}$, gekoppelt an PAB-Cellulose, durch. Mit dest. Wasser eluierten wir eine Substanz, die keine Schultz-Dale-Reaktion zeigte. Durch Abspaltung mit 0,1M Glycinpuffer bei pH 2,5 erhielten wir das an Anti D absorbierte Protein, das wir durch Sephadex-G 10-Passage von dem Glycinpuffer abtrennten. Koppelten wir Anti D-freies IgG anstelle von IgG Anti D mit PAB-Cellulose, dann wurde bereits mit dest. Wasser das gesamte Ausgangsprodukt eluiert. Die aus rh-negativen Erythrocyten isolierten kleinmolekularen Proteine konnten mit Anti D-PABCellulose nicht adsorbiert werden. Die Gesamtausbeute des durch das Immunadsorbens isolierte und mit dem Glycinpuffer wieder abgetrennte Protein betrug nach 


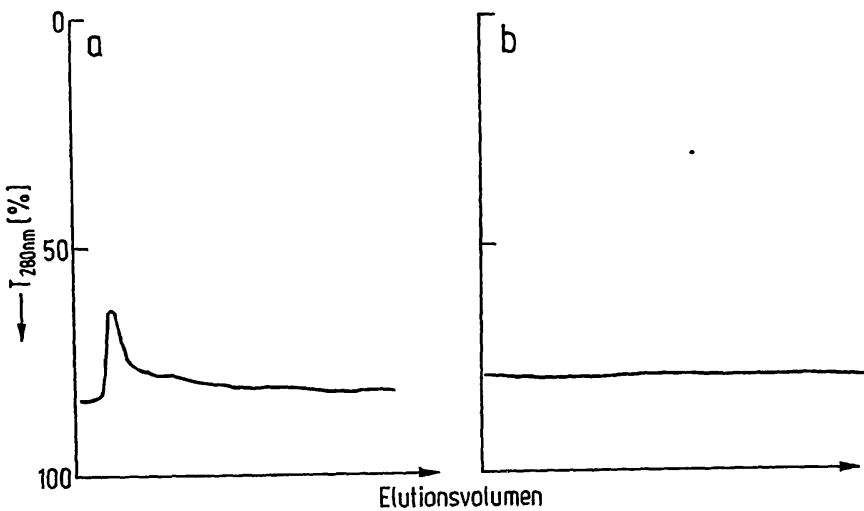

Abb. 5

Immunadsorption nach Kopplung von gereinigtem Anti $\mathrm{D}$ an PABCellulose durch Diazotierung. Saule $30 \times 1 \mathrm{~cm}$. Elution mit Wasser 作 geschwindigkeit $60 \mathrm{ml} / \mathrm{Std}$. Registrierung: Uvicord $\lambda=280 \mathrm{~nm}$

a) Fraktion 1 des Diffusates I/II aus dem Hämolysat Rh-positiver Erythrocyten nach Sephadex-Gelfiltration über $C$
Blutgruppe $0 \mathrm{Rh}+$ (CcDee) $M$

b) Fraktion I des Diffusates I/II aus dem Hämolysat rh-negativer Erythrocyten. Konserve 161, Blutgruppe A rh- (ccdee) $\oplus$

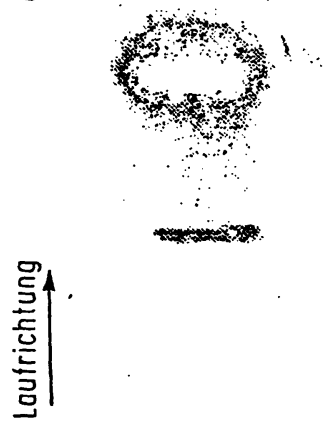

$\Theta$

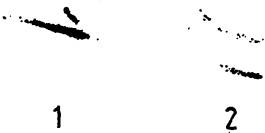

Abb. 6

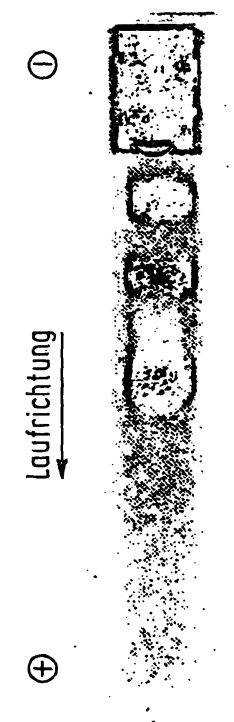

1

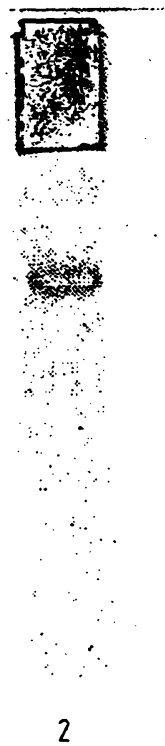

Abb. 7

Abb. 6

Cellulose-Acetat-Elektrophorese. Puffersystem: Natrium-Veronal- Salzsäure pH 8,6 Laufzeit 120 Min. Stromstärke 3-5 mA.

1. Fraktion I vor Anti D-Immunadsorption, 2. Fraktion I nach Anti D-Immunadsorption

$$
\text { Abb. } 7
$$

Polyacrylamidelektrophorese mit 7,5proz. Trenngel. Trispuffer pH 8,2 Laufzeit $90 \mathrm{Min}$. Stromstärke $3 \mathrm{~mA}$ pro Gelzylinder. 1. Fraktion I vor Anti D-Immunadsorption, 2. Fraktion I nach Anti

Entsalzen an Sephadex G $102-3 \mathrm{mg}$, gewonnen aus $200 \mathrm{ml}$ gepackter Rh-positiver Erythrocyten (Abb. 5). Dieses Protein war sowohl auf Acetatcellulose-Folie als auch in der diskontinuierlichen Polyacrylamid-Elektrophorese und in dem Fingerprint-Verfahren einheitlich (Abb. 6, 7). Der Stickstoffgehalt betrug 14,6\%. Das IR-Spektrum war für Peptide spezifisch (Abb. 8). Das Molekulargewicht des diffusiblen Proteins lag nach Gelfiltration mit Vergleichssubstánzen Protaminsulfat (MG 5-6000) und Ribonuclease (13-14000) um 10000. Nach Hydrolyse des Proteins mit 6N Salzsäure 24 Stdn. bei $110^{\circ}$ wurden dünnschichtchromatographisch 14 Aminosäuren nachgewiesen, unter denen die Flecken für Alanin, Asparaginsäure und Lysin am intensivsten waren (Abb. 9). Das Ergebnis dieser zweidimensionalen Aminosäurechromatographie konnte durch die quantitative Aminosäurebestimmung mit gaschromatographischer Trennung der TrifluoracetylAminosäurebutylester und durch die AminosäureAnalyser-Untersuchungen bestätigt werden. Die Aminosäure-Untersuchungen zeigten, daß Alanin die höchste

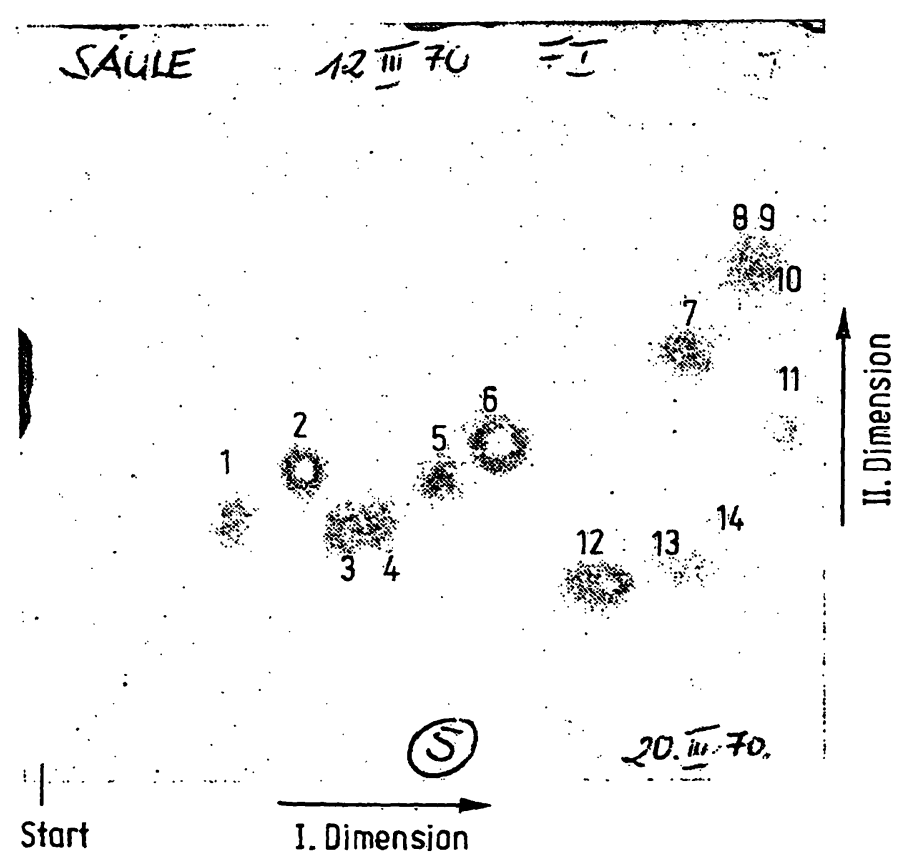

Abb. 9

Zweidimensionale Dünnschichtchromatographie auf $7,5 \times 7,5 \mathrm{~cm}$ Platten nach Hydrolyse des Anti D-hemmenden Proteins 24 Stdn. be $120^{\circ}$ in $6 \mathrm{~N} \mathrm{HCl}$. Laufmittel: I. Dimension Butanol: Eisessig: Wasser = 12:3:5 (v/v/v). II. Dimension Phenol: Wasser: Ammoniak $160: 36: 0,9(\mathrm{v} / \mathrm{v} / \mathrm{v})$ 1. Asp, 2. Glu, 3. Ser, 4. Gly, 5. Thr, 6. Ala, 7. Val, 8. Leu, 9. Ile,
10. Phe, 11. Pro, 12. Lys, 13. Arg, 14. His
Abb. 8

IR-Spektrum des Anti D-hemmenden Proteins, aufgenommen als $\mathrm{KBr}$-Preßling mit dem Gerät von Perkin Elmer

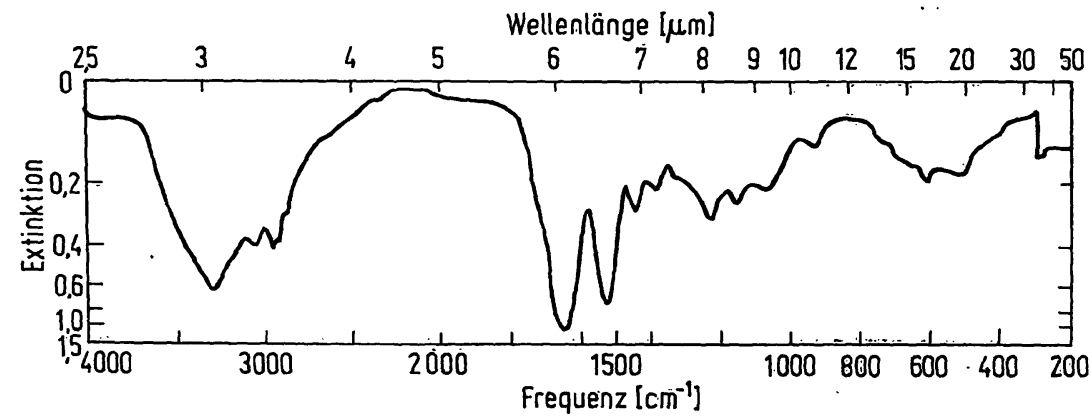

Z. klin. Chem. u. klin. Biochẹm. / 9. Jahrg. 1971 / Heft 5 
Abb. 10

Gaschromatographie der Trifluoracetyl-Aminosäure-Butylester nach Hydrolyse des Anti D-hemmenden Proteins (24 Stdn., 120 $)$ in $6 \mathrm{~N} \mathrm{HCl}$. Glassäule $180 \times 0,3 \mathrm{~cm}, 1,5$ proz. OV 17 Chromosorb G-HP 100-120 mesh

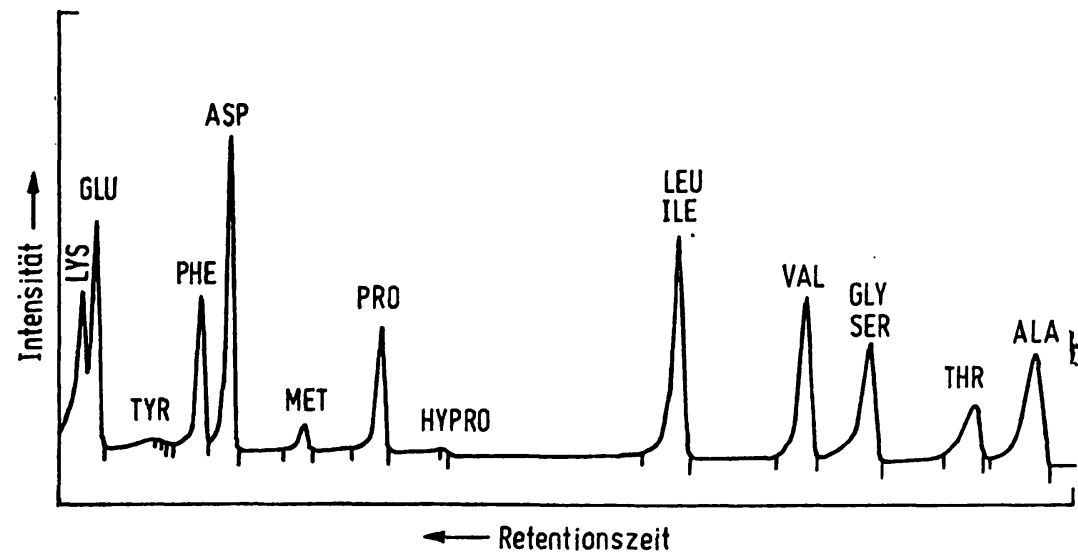

Tab. 2

Aminosăureverteilung in Mol\% der Fraktion I des Diffusates $1 / 11$ von Rh-positiven und rh-negativen Erythrocyten

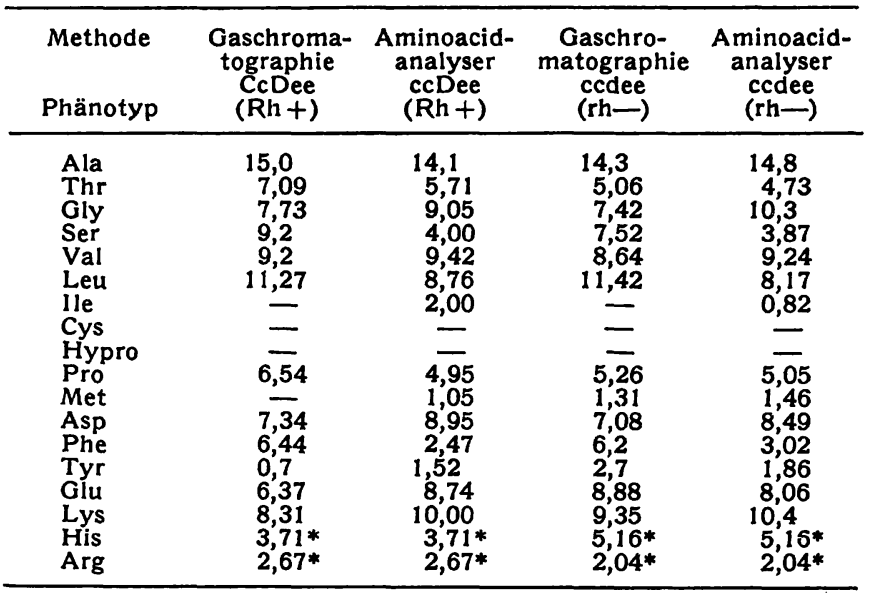

* Histidin und Arginin konnten gaschromatographisch nicht untersucht werden, da keine quantitative 'Derivat-Bildung stattfand.

Konzentration unter, den neutralen, Asparaginsäure unter den sauren und Lysin unter den basischen Aminosäuren besitzt (Abb. 10). Aus Tabelle 2 ist zu entnehmen, daß Leucin bei den Proteinen von Rh-positiven Erythrocyten erhöht und Histidin vermindert ist gegenüber den Proteinen, erhalten von rh-negativen Erythrocyten.

Die quantitative Ausbeute der proteinhaltigen Sephadex-Fraktion differierte bei Erythrocyten verschiedener Rh-Phänotypen und entsprach den quantitativen Differenzen der D-Rezeptoren-Anzahl, die von RochNa und Hughes-Jones (20) mit markiertem $\gamma$-Globulin bei den einzelnen Rh-Phänotypen ermittelt worden war (Tab. 3).
Detaillierte Darstellung der Antigen-Charakterisierung durch den Schultz-Dale-Test nach aktiver und passiver Immunisierung von Meerschweinchen soll in einer zweiten Mitteilung abgehandelt werden.

\section{Diskussion}

$\mathrm{Da}$ seit der serologischen Charakterisierung des $\mathrm{Rh}$ Systems zahlreiche chemisch unterschiedliche Bestandteile der Erythrocytenmembran als Rh-Rezeptor beschrieben worden waren, deren Antigen-Eigenschaften nicht sicher bewiesen werden konnten, erschien es uns für die Antigen-Isolierungstechnik und den AntigenNachweis notwendig, eine neue Versuchskonstellation zu wählen. Der Hämagglutinations-Hemmtest zum D-Antigen-Nachweis kann durch eine Reihe von Faktoren unspezifisch beeinflußt werden, so daß z. B. durch Lecithin oder durch Mineralien in hoher Konzentration - Substanzen, die bei der Erythrocytenaufarbeitung in beträchtlicher Konzentration anfallen eine Anti D-Hemmung vorgetäuscht werden kann. In der oben geschilderten Versuchsanordnung gelang es uns, nach Hämolyse des Erythrocyten und durch erschöpfende Dialyse des nativen Hämolysates und Gelfiltration des Diffusates die aus den Erythrocyten freigesetzten Mineralien und kleinmolekularen organischen Substanzen zu entfernen. Nach Gefriertrocknung, Homogenisieren und Eliminierung der Mineralien war die Stabilität des Stromaverbandes reduziert, so daß ein kleinmolekulares Protein durch erneute Dialyse aus dem Stroma isoliert werden konnte. $\mathrm{Da}$ wir bei Kühlraumtemperatur arbeiteten und nach der Dialyse dieses Proteins sofort gefriergetrocknet

Tab. 3

Ausbeute der kleinmolekularen Proteinfraktion in $\mathrm{mg}$ pro $100 \mathrm{mg}$ Diffusat $\mathrm{I} / \mathrm{II}$ nach Sephadex-G 10-Gelfiltration bei verschiedenen Rh-Phänotypen im Vergleich zu der D-Rezeptorenanzahl pro Erythrocyt bei verschiedenen Rh-Phänotypen

\begin{tabular}{|c|c|c|c|c|}
\hline Phänotyp & Genotyp & Anzahl der Proben $\mathbf{n}$ & $\begin{array}{l}\text { Kleinmolekulare Protein- } \\
\text { fraktion des Hämolysates in } \\
\mathrm{mg} / 100 \mathrm{mg} \text { Diffusat } \pm 5 \%\end{array}$ & $\underset{\text { Erythrocyt* }}{\text { Rezeptorenanzahl pro }}$ \\
\hline $\begin{array}{l}\text { CcDee } \\
\text { ccDee } \\
\text { ccDEe } \\
\text { CCDee } \\
\text { CcDEe } \\
\text { ccDEE }\end{array}$ & $\begin{array}{l}\text { CDe/cde }\left(R_{1} r\right) \\
\text { cDe/cde }\left(R_{0} r\right) \\
\text { cDE/cde }\left(R_{2} r\right) \\
C D e / C D e\left(R_{1} R_{1}\right) \\
C D e / c D E\left(R_{1} R_{2}\right) \\
\text { CDE/CDE }\left(R_{2} R_{2}\right)\end{array}$ & $\begin{array}{r}11 \\
5 \\
2 \\
2 \\
4 \\
2\end{array}$ & $\begin{array}{l}6,7 \\
16 \\
8,1 \\
16,2 \\
23 \\
17\end{array}$ & $\begin{array}{r}9900-14600 \\
12000-20000 \\
14000-16000 \\
14500-19300 \\
23000-31000 \\
15800-33000\end{array}$ \\
\hline
\end{tabular}

* Ermittelt von E. RochNa und N. C. Hughes-Jones (20). 
wurde, ist ein Spaltprodukt durch proteolytische Membran- oder Bakterienenzyme unwahrscheinlich, um so mehr, da wir bei 100 Vergleichsuntersuchungen sowohl in bezug auf die quantitative Ausbeute als auch auf die chemische Zusammensetzung gut reproduzierbare Isolierungsprodukte erhielten. Die D-AntigenEigenschaften dieses Proteins wurden in dem SchultzDALE-Test nach aktiver und passiver Immunisierung mit Anti D in einer großen Versuchsreihe geprüft. Obwohl dieses Protein nach Gelfiltration an Sephadex und Ionenaustauscherchromatographie einheitlich erschien, konnte es durch Polyacrylamid-Disk-Elektrophorese noch in mehrere Fraktionen getrennt werden. Wir führten deshalb zur spezifischen Isolierung der für die Antigeneigenschaften verantwortlichen Proteinkomponente eine Anti D-Immunadsorption durch. Nach Kopplung von gereinigtem Anti D mit PABCellulose durch Diazotierung konnten wir ein Protein absorbieren, das von dem Antikörper durch 0,1M Glycinpuffer bei $\mathrm{pH} 2,5$ freigesetzt wurde. Dieses Protein hatte ein Molekulargewicht um 10000. Der Proteincharakter konnte bei einem Stickstoffgehalt von $14,6 \%$ durch die stark positive Biuret-Reaktion bei geringer Chromogenbildung mit Ninhydrin-Reagenz und vor allem durch das IR-Spektrum bewiesen werden. $\mathrm{Da}$ es bei den Untersuchungen mit Fingerprint, Diskelektrophorese und zweidimensionale Chromatographie einheitlich war, kann ein weitgehend homogenes Protein angenommen werden. Nach saurer $\mathrm{Hy}-$ drolyse fanden wir mit der Dünnschichtchromatographie, der Gaschromatographie, der TrifluoracetylAminosäurebutylester und mit dem Aminosäure-Analyser 14 Aminosäuren. Aufgrund des niedrigen Molekulargewichtes um 10000 und des hohen Gehaltes an Lysin (etwa 10\%) unterscheidet es sich von anderen bekannten Serum- und Erythrocytenmembranproteinen. Kolorimetrische und gaschromatographische Untersuchungen ergaben keinen Anhalt für einen Kohlenhydratanteil. Cholesterin oder Triglyceride waren nicht. nachweisbar. Lecithin konnte chromatographisch ausgeschlossen werden. Die Deutung des geringen Lipidphosphorgehaltes von $0,1-0,2 \%$ ist noch Gegenstand der z. Z. laufenden Untersuchungen. Die Ausbeute des Proteins von 2-3 mg/l Blut entspricht unter Zugrundelegung eines Molekulargewichtes von 10000 der Menge, die aufgrund der Anzạhl von D-Rezeptoren, ermittelt durch die Anti D-Markierung an Erythrocyten, zu erwarten war (21). Dieser Befu'nd gewinnt an Bedeutung, da auch die unterschiedlich große Proteinausbeute bei Erythrocyten mit verschiedenen $\mathrm{Rh}$ Phänotypen mit den von RochNa und Hugres-Jones (20) ermittelten Differenzen in der $\mathrm{Rh}$-Rezeptorenanzahl bei den einzelnen Phänotypen eine gute Korrelation zeigte. Auch die unterschiedliche Aminosäurerelation der durch Gelfiltration isolierten Fraktion bei rh-negativen und Rh-positiven Erythrocyten könnte auf eine proteinabhängige Antigen-Spezifität hinweisen. Die Charakterisierung der D-Antigen-Eigenschaften wurde mit der SchulTz-DALE-Reaktion nach aktiver und passiver Immunisierung durchgeführt und soll in einer gesonderten Mitteilung dargestellt werden. Unabhängig von der hier erwähnten D-Antigenfunktion dürfte diesem kleinmolekularen Protein jedoch auch eine Bedeutung in der Struktur der Erythrocytenmembran zukommen.

\section{Danksagung}

Herm Dr. RoelCKe, Immunologisches und Serologisches Institut der Universität Heidelberg, danken wir für die Durchführung der zahlreichen serologischen Untersuchungen und seine wertvolle Beratung. Herrn Dr. K. Walsh, Department für Biochemie, Seattle/USA, möchten wir für die Durchführung der Aminosäuren-Analysen mit dem Aminosäure-Analysator danken, Herrn Dr. W. Otring, Max Planck-Institut Heidelberg, für die Anfertigung und Beurteilung der IR-Spektren, Herrn Dr. A. KLUGE sind wir für die großzügige Überlassung der 180 Spenderkonserven zu großem Dank verpflichtet. Fräulein G. JENSSEN und Fräulein G. SOMMER danken wir besonders für die gewissenhafte Durchführung der zahlreichen Untersuchungen.

\section{Literatur}

1. Brown, G. L. und A. K. Brown, Symp. Soc. Exp. Biol. XII, 6, Univ. Press, Cambridge (1958). - 2. Grubhofer, N. und L. SChrerth, Hoppe-Seyler's Z. physiol. Chem. 297, 108 (1954). 3. Avrameas, St., B. Taudou und S. Chuilon, Immunochemistry 6, 67 (1969). - 4. RoelCKe, D. und H. Jungfer, Klin. Wschr. 48, 752 (1970). - 5. StahL, E., Dünnschichtchromatographie, Springer-Verlag Berlin-Göttingen-Heidelberg (1962). 6. Zinzadze, C., Ind. Eng. Chem. Anal. Ed. 27, 24 (1935). 7. MAURER, H. R., Disk-Elektrophorese. Walter de Gruyter \& Co., Berlin (1968). - 8. WrNzLER, R. J., Meth. Biochem. Analysis 2, 279 (1955). - 9. Drsche, Z. und L. B. Shetries, J. biol. Chemistry 175, 595 (1948). - 10. SVennerholm, L., Biochim. biophysic Acta (Amsterdam) 24, 604 (1957). - 11. Warren, L., J. biol.
Chemistry 234, 1971 (1959). - 12. Cessi, C. und F. Piliego, Biochem. J. 77, 508 (1960). - 13. Fiske, C. H. und Y. Subarrow, J. biol. Chemistry 66, 375 (1925). - 14. Pearson, S., S. Stern und T. H. McGavack, Analytic. Chem. 25, 813 (1953). - 15. Bradstreet, R. B., Analytic. Chem. 26, 185 (1954). - 16. Gehrke C. W. und L. D. Stalling, Separation Science 2/11101 (1967). 17. Metz, J., F. W. Ebert und H. Weicker, im Druck. - 18. Grässlin, D. und H. Wercker, Clin. chim. Acta (Amsterdam) 21, 15 (1968). - 19. YANG, I. und S. Hakomori, J. biol. Chemistry, im Druck. - 20. Rochna, E. und N. C. Hughes-Jones, Vox Sang. 10, 675 (1965). - 21. Rosenpiend, R. E. und S. KochwA, J. Immunol., Baltimore 92, 693 (1964).
Prof. Dx. H. Weicker 69 Heidelberg Hospitalstr. 3 